\title{
Application of PET/CT in Oncologic Imaging
}

\author{
Sohaila Yazdani, ${ }^{1,}$ Homayon Fallah, ${ }^{1}$ and Fahimeh Rezaie ${ }^{1}$ \\ ${ }^{1}$ Kermanshah University of Medical Science, Kermanshah, Iran \\ "Corresponding author: Sohaila Yazdani, Kermanshah University of Medical Science, Kermanshah, Iran. E-mail: sohailayazdani@gmail.com
}

Received 2016 December 21; Accepted 2017 February 08.

\begin{abstract}
Background: There are many challenges in oncologic imaging including: more accurate staging, determine site with highest probability of tumor existence for biopsy, especially for brain tumors because of the sensitive anatomical position, determine the exact location of the tumor for radiotherapy treatment planning and etc. Positron emission tomography (PET) is imaging modality which provides some special functional information. The basis of the PET system is detection of tow photons which are generated by positron/electron encounter which occurs directly after positron emission by radionuclide. However, it is powerless in providing anatomical information. Computed tomography (CT) generates three dimensional reconstructions of patients anatomy based on differences in X-ray attenuation. Combination of PET and CT is able to provide anatomical and physiological information of patients synchronizes.

Objectives: In this paper, we evaluate application of PET/CT for improve oncologic imaging.

Methods: The papers were searched in PubMed and Scopus databases with the relevant key words including, PET/CT, Oncologic Imaging and Cancer staging.

Results: The most relevant biomarker for PET is 8F- FDG (F-18 Fluorodeoxyglucose), which is incorporated into malignant cells by glucose transporters which are over expressed by cancer cells. CT is the common imaging modality in diagnosis the cancers. It is due to its speed, high special resolution and relative cost-effectiveness. The combination of these two modalities in one system produced PET/CT system which is more accurate than these two imaging modalities separately and enhances the sensitivity and specificity of diagnosis. For example in a study which used PET/CT for differentiation between the malignant and benign disease Presacral, sensitivity, specificity, the positive predictive value and the negative predictive value are $100 \%, 96 \%, 88 \%$ and $100 \%$ respectively. In another study, which was done for preradiotherapy staging of head-and-neck SCCs, it was shown that FDG-PET/CT detected 17 of 17 heminecks and 26 of 27 nodal zones histologically positive by dissection (100\% and $96 \%$ sensitivity, respectively).

Conclusions: PET/CT can provide diagnosis and more accurate staging, location of tumor with its capability in providing the anatomical and physiological information synchronizes. Therefore PET/CT plays an important role in oncology.
\end{abstract}

Keywords: PET/CT, Oncologic Imaging and Cancer Staging

This is an abstract presented in the 33rd Iranian congress of radiology (ICR) and the 15th congress of Iranian radiographic science association (IRSA). 